BMJ Open

Sport \&

Exercise

Medicine

\section{Unloading knee brace is a cost-effective method to bridge and delay surgery in unicompartmental knee arthritis}

To cite: Lee PYF, Winfield TG, Harris SRS, et al. Unloading knee brace is a cost-effective method to bridge and delay surgery in unicompartmental knee arthritis. BMJ Open Sport Exerc Med 2017;2:e000195. doi:10.1136/bmjsem-2016000195

- Prepublication history is available. To view please visit the journal (http://dx.doi.org/ 10.1136/bmjsem-2016000195).

Accepted 31 January 2017

CrossMark

${ }^{1}$ South Wales Orthopaedics Research Network Welshbone, Cardiff, UK ${ }^{2}$ Department of Orthopaedics \& Trauma, Abertawe Bro Morgannwg University Health Board, Bridgend, UK ${ }^{3}$ Department of Medical engineering, Cardiff University, Cardiff, UK ${ }^{4}$ Swansea Centre for Health Economics, Swansea University, Swansea, UK

Correspondence to DrPaul YF Lee; leepyf@ cardiff.ac.uk

\section{ABSTRACT}

Background: Unloading knee braces can provide good short-term pain relief for some patients with unicompartmental osteoarthritis (UOA). Their cost is relatively small compared with surgical interventions. However, no previous studies have reported their use over a duration of 5 years or more.

Methods: Up to 8 years of prospective data were collected from 63 patients who presented with UOA. After conservative management with analgesia and physiotherapy, patients were offered an unloading brace. EQ-5D (EuroQol five dimensions) questionnaires were collected at baseline and after wearing the brace. Cost and quality-adjusted life years (QALYs) were compared with a total knee replacement (TKR) with an 8-month waiting duration and 8 years of results.

Results: Patients experienced a mean increase in EQ$5 \mathrm{D}$ of 0.42 with an average duration of wear of 26.1 months resulting in an increase of 0.44 in QALYs with a mean cost of $£ 625$. The adoption of an unloader knee brace was found to be a short-term cost-effective treatment option with an 8-month incremental cost effectiveness ratio of $£ 9599$. Compared with no treatment, the unloader knee brace can be considered cost effective at 4 months or more. At 8 years followup, the unloader knee brace demonstrated QALYs gain of 0.43 and with an incremental cost-effectiveness ratio of - $£ 6467$ compared with TKR.

Conclusion: Unloading knee braces are cost effective for the management of UOA. These findings strongly support the undertaking of further research into the long-term impact of unloading knee brace. The unloader knee brace has benefits to the National Health Service for capacity, budget, waiting list duration, frequency of surgery and reducing the required severity of surgical intervention.

\section{INTRODUCTION}

Knee osteoarthritis is a prominent issue in the UK with $18 \%$ of the population aged 45 or over seeking treatment. ${ }^{1}{ }^{2}$ Total knee replacement (TKR), unicompartmental knee replacement (UKR) and high tibial osteotomy (HTO) are techniques that have been used to treat unicompartmental osteoarthritis (UOA). These procedures are commonly found to be highly cost-

\section{What is known about this subject?}

Unloading knee braces can provide good short-term pain relief for some patients with unicompartmental osteoarthritis. There have been no studies reporting the health economics of their use as bridging therapy for surgery and also long-term use over 5 years.

\section{What this study adds to existing knowledge?}

- This study suggests that unloading knee brace is a cost-effective method as a bridging therapy and its long-term use can delay and reduce surgical intervention.

- This study suggests a minimum cost-effective period (4 months) as well as the most beneficial duration (7-12 months) of using the unloading brace.

- This study provides evidence that if patients wear the brace for more than 2 years, surgical intervention is not needed.

effective. $^{3}{ }^{4}$ It has been well documented that UOA is caused by an increase in force through one compartment due to malalignment of the joint. $^{2}$ As a complementary technology to surgical intervention, patients with UOA may adopt an unloading knee brace. Biomechanically, the brace exerts a valgus or varus force dependent on the affected compartment to reposition the damaging force away from the arthritic area and reduce pain. Therefore, the unloading brace may delay the need for a patient to undergo surgery. The Patient Episode Database for Wales (PEDW) presents mean waiting times for some local health boards of a primary TKR in excess of 8 months. ${ }^{5}$ Instead, adopting a knee brace may benefit the individual during the waiting period, improving stability within the knee, 
consequently shortening waiting list times through reduction in need for surgical intervention. Additionally, knee braces could have positive budget impact effects and potential capacity benefits over surgeries.

Evidence suggests that unloading knee braces are a safe treatment option for unicompartmental arthritis, which can reduce pain and increase activity in the short term, but their effect in the long term is uncertain. ${ }^{6} 7$ In the literature, follow-up for longer than 3 years has not been evaluated. A Cochrane review showed that there was limited high-quality evidence over 12 months to assess the overall effect of the brace and found the brace's advantages inconclusive in shortterm studies. ${ }^{8}$

This study aims to use 8 years of follow-up data to investigate the short-term and long-term cost-effectiveness of the unloader knee brace compared with no treatment while on the waiting list and TKR at 8 years.

- Does the unloading knee brace delay surgical intervention, such as TKR, for UOA?

- Is the use of the unloading knee brace for treatment of UOA cost-effective? For the cost utility analysis, the initial evaluation will focus on the short-term benefits of the unloader knee brace, ${ }^{9}$ viewing the technology compared with the average waiting list duration.

- What is the long-term cost-effectiveness of the unloader knee brace when compared with the cheapest and most effective surgical option-TKR?

\section{METHODS}

The study considers 63 consecutive prospective patients with end-stage unicompartmental knee osteoarthritis (lateral or medial) who attended a district general hospital in Wales, UK, between August 2007 and February 2009, and were prescribed the unloader knee brace while they were on the waiting list for surgery. Patients with tricompartmental or patellofemoral osteoarthritis were excluded from the study, as were those who had previously undergone joint replacement surgery. The local health board approved this analysis.

At the time of fitting, patients were instructed by a trained nurse practitioner how to fit the brace at home. Patients completed the EQ-5D-3L ${ }^{10}$ Questionnaire before the brace was fitted, and then subsequently when either the patient ceased using the brace or at the end of the study period in 2015. These responses consider the change in a patients' mobility, pain, activity, anxiety and the ability to self-care and be independent. These were validated via time trade-off and standard gamble approaches to offer UK index quality of life scores.

Figure 1 presents the typical treatment pathway for patients receiving a knee brace for UOA including unit costs available from the Personal Social Services Research Unit 2015. ${ }^{11}$ At 2015 values, the mean cost of administering the unloader knee brace, including the cost of the brace and associated clinician time, was calculated to be $£ 625 .{ }^{11}$ Costs ranged from $£ 592$ for those patients who were discharged without experiencing any problems following nurse led clinic appointment for any necessary adjustments to 9989 for those requiring multiple referrals to consultant led clinics with X-rays of the knee. ${ }^{11}$ Patients with duration of wear of less than 3 months did not incur any costs associated with adjustment of the brace. The costing approach accounts for the distinction between patient contact hours and general working hours.

The statistical approach follows a cost utility analysis (CUA) framework which evaluates the costs and quality-adjusted life years (QALYs) of the unloader knee brace compared with the current treatment approach, in this case, TKR. The comparison is done on a patient by patient basis; results are offered as an average of the observed cohort. The comparator data are sourced from a TKR study ${ }^{12}$ with three additional assumptions. First, TKR has an effective duration of 20 years, a figure quoted by the National Health Service guidelines. Second, the benefits of TKR have been exponentially extrapolated beyond the study's reported 5 -year end point to correspond with the maximum duration of wear for the knee brace. This approach utilises the trend component from the observed data and extends this beyond the study period. Finally, EuroQol five dimension (EQ-5D) values are assumed constant for patients that are awaiting treatment. All statistical approaches were undertaken using a combination of MS Excel and STATA V.13.

The short-term CUA views the unloader knee brace compared with the PEDW All Wales mean waiting time of primary TKR of 8 months, ${ }^{5}$ currently at 18 months in our hospital. For this approach, the costs and benefits of the unloader knee brace are compared with no treatment. To analyse QALYs, the area under the curve approach is used. A QALY is defined as the arithmetic product duration and quality of life, with the latter measured by the EQ-5D index score. The long-term comparison to TKR evaluates the complete data duration of the unloader knee brace compared with the costs and benefits that each individual would have experienced in the normal pathway. The comparator approach includes an 8-month waiting time which applies the cost and benefits on a monthly basis. Therefore, the overall cost of a TKR is divided by 240 (20 years of 12 months). The TKR comparator is characterised by high initial costs, given the disparity in duration with the unloader knee brace, it is deemed necessary to linearly apportion TKR costs. Incremental cost-effectiveness ratios (ICER) were calculated for both short-term and long-term approaches. Descriptive results are calculated for the delay and frequency of surgical intervention.

A discounting rate of $3.5 \%$ was applied, as recommended by the National Institute for Health and Care Excellence (NICE) guidelines 2013. ${ }^{13}$ Aside from the 
Figure 1 Unloader knee brace patient pathway. *Nurse costs defined as patient contact costface-to-face at $£ 147$ per hour. **Doctor costs defined as general contract cost-admin/ordering the brace $£ 106$ per hour.

${ }^{\star * \star}$ Consultant cost defined as patient contact cost-face-to-face at $£ 331$ per hour.

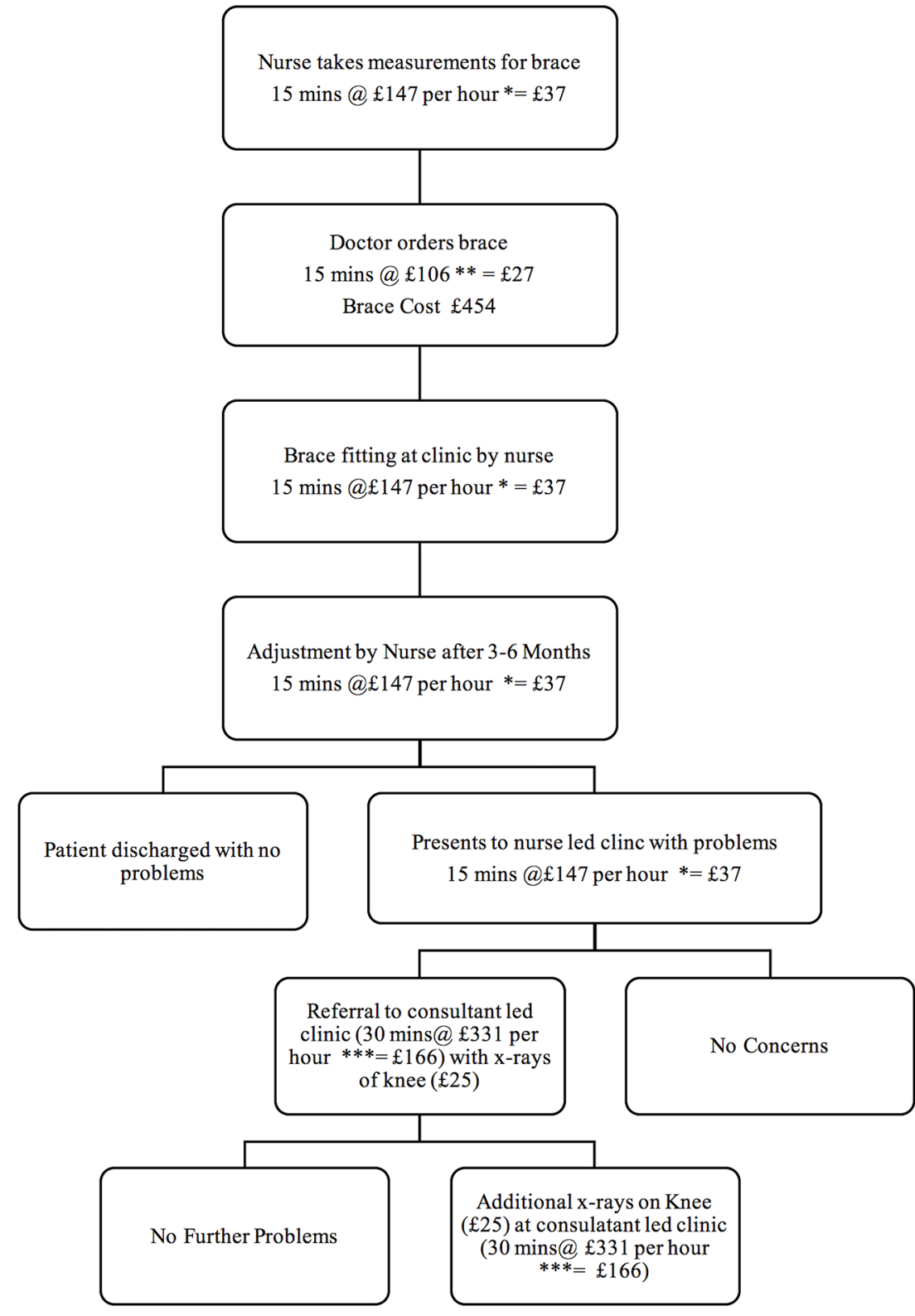

extrapolation method used for the TKR comparator, no missing data techniques were required. One-way sensitivity analyses were undertaken alongside bootstrapping methods to offer cost-effectiveness accessibility curves for both short-term and long-term analysis. Additionally, a threshold analysis was used to identify the duration (in months) at which the unloader knee brace became cost-effective at a $£ 30000$ ICER.

\section{Results}

Sixty-three patients were involved in the study, no patients were lost to follow-up; however, one patient withdrew from the study but had not had surgery at final follow-up. Descriptive statistics concerning patient demographics and baseline characteristics are presented in table 1 below:

While the cohort has a similar gender profile to Dakin et $a l^{12}$ with an approximate 1:1.4 males to females, the population is considerably younger with mean age of 50.9 compared with 71 years. The mean duration that patients wore the unloader brace was approximately 26 months, considerably longer than the mean waiting time for TKR presented by PEDW. The average unloader knee brace duration of wear was longer than the current 8 months waiting list as some patients opted out of surgery, preferring continued use of the unloader knee brace. Excluding the 25 patients who did not require surgical intervention after wearing the brace at final follow-up, a mean duration of wear of 8.6 months is observed. A linear regression established that there were no significant differences in duration of wear attributed to gender, age, BMI, socioeconomic group, leg or compartment.

Surgical interventions were required for 38 patients, of whom $50 \%$ required TKR, $37 \%$ had UKR and $13 \%$ had HTO. Medial compartmental osteoarthritis accounted for $73.8 \%$ of unsuccessful patients. In this population, patients wore the brace for an average of 8 
Table 1 Baseline characteristics

\begin{tabular}{|c|c|c|c|c|c|}
\hline \multicolumn{3}{|c|}{$\begin{array}{c}\text { Mean } \\
\text { (SD) }\end{array}$} & \multicolumn{2}{|l|}{ Min } & \multirow[t]{2}{*}{ Max } \\
\hline Age (years) & \multicolumn{2}{|l|}{$50.9(9.7)$} & 34 & 81 & \\
\hline $\mathrm{BMI}$ & \multicolumn{2}{|l|}{$28.6(9.2)$} & 20.6 & \multicolumn{2}{|l|}{38.6} \\
\hline Duration of wear (months) & \multicolumn{2}{|l|}{$26.1(31.6)$} & 1 & \multicolumn{2}{|l|}{96} \\
\hline Baseline EQ-5D & \multicolumn{2}{|l|}{$0.012(0.194)$} & -0.371 & \multicolumn{2}{|l|}{0.656} \\
\hline Gender & \multicolumn{3}{|c|}{ Leg } & \multicolumn{2}{|c|}{ Compartment } \\
\hline Male & $26(41.3 \%)$ & Left & $36(57.1 \%)$ & Lateral & $19(30.2 \%)$ \\
\hline Female & $37(58.7 \%)$ & Right & $27(42.9 \%)$ & Medial & $44(69.8 \%)$ \\
\hline$n=63$ & & \multicolumn{2}{|c|}{ Socioeconomic groups } & \multicolumn{2}{|c|}{ Return to work with the use of brace } \\
\hline Professionals & & \multicolumn{2}{|l|}{4} & \multicolumn{2}{|l|}{4} \\
\hline Office based & & \multicolumn{2}{|l|}{21} & \multicolumn{2}{|l|}{20} \\
\hline Heavy manual & & \multicolumn{2}{|l|}{20} & \multicolumn{2}{|l|}{18} \\
\hline Light manual & & \multicolumn{2}{|l|}{13} & \multicolumn{2}{|l|}{13} \\
\hline Unemployed & & \multicolumn{2}{|l|}{5} & \multicolumn{2}{|l|}{$n / a$} \\
\hline
\end{tabular}

EQ-5D, EuroQol five dimensions.

months, ranging from 1 to 24 months, with $40.5 \%$ ceasing to wear the brace within the first 6 months from prescription. At 24 months, 16 (25.4\%) patients from the cohort of 63 were still wearing the unloader knee brace. The Kaplan-Meier curve (figure 2) shows that if patients' symptoms were still tolerable at 24 months, the chances of undergoing subsequent surgery declined significantly. A success was defined as a patient avoiding surgery by wearing the brace. Where patients wore the brace for 6 months rather than 3 months, the proportion of success doubled from $4.1 \%$ to $8.3 \%$. The average duration of wear in the success group is 46.5 months longer than the average wear in the unsuccessful group.

Table 2 demonstrates positive changes in utility and QALY gains for all patients and for each duration of wear subgroup. QALY gains are positively correlated with the duration of wear. For all patients, mean EQ-5D utility substantially increased from 0.01 at baseline to 0.4 at follow-up. Similar increases are observed across the duration of wear sub-groups, with the largest mean increase observed for patients with duration of wear of 7-12 months. All patients who wore the knee brace for 25 months or more and opted to continue to use the knee brace are characterised by the largest QALY gains.

\section{Short-term effects}

Assuming that patients wore the knee brace for the entire TKR mean waiting list duration of 8 months, there are potential QALY gains as demonstrated by table 3 below:

Table 3 reports the relative costs and QALYs gained for the unloader knee brace compared with TKR; these are used to calculate an ICER, defined as the ratio of the change in costs compared with incremental benefits of the intervention. Results from table 3 indicate that at a cost of £625, wearing an unloader knee brace for the entire TKR mean waiting list duration of 8 months offers patients a QALY gain of 0.07 ; this provides an ICER of £9599, substantially below the $£ 20000$ $£ 30000$ per QALY gained range currently deemed acceptable by NICE. One-way sensitivity analyses, considering a $30 \%$ increase, in the direct cost of the unloader knee brace, increases the ICER to $£ 11688$, While a $30 \%$ decrease in the direct cost reduces the ICER to $£ 7511$. These values remain within the NICE acceptability range. Figure 3 demonstrates the time variability of the ICER for the unloader knee brace compared with an alternative of no treatment.

\section{Long-term effects}

QALY gains are also observable for the longer term, with the results presented in table 4 below:

The results presented in table 4 demonstrate that at a cost of $£ 625$, the unloader knee brace provides a QALY gain of 0.44. In comparison, given a mean waiting time of 8 months, and the self-selection of treatment alternatives, the TKR provides lower QALYs at higher costs. Consequently, the unloader knee brace dominates surgical intervention with an ICER of $£ 6467$. One-way sensitivity analyses, considering a 30\% reduction, in the product cost of the knee brace, increases the cost-effectiveness with an ICER of $£ 13$ 670. In contrast, a $30 \%$ increase in the product cost produces an ICER of $£ 630$. While the off-loader knee brace no longer dominates surgical intervention, 
Figure 2 Kaplan-Meier survival curve for unloader braceKaplan-Meier survival analysis demonstrated rapid conversion to TKR for the first 12 months, which then tails off to show no conversion after 24 months of using the unloader knee brace.

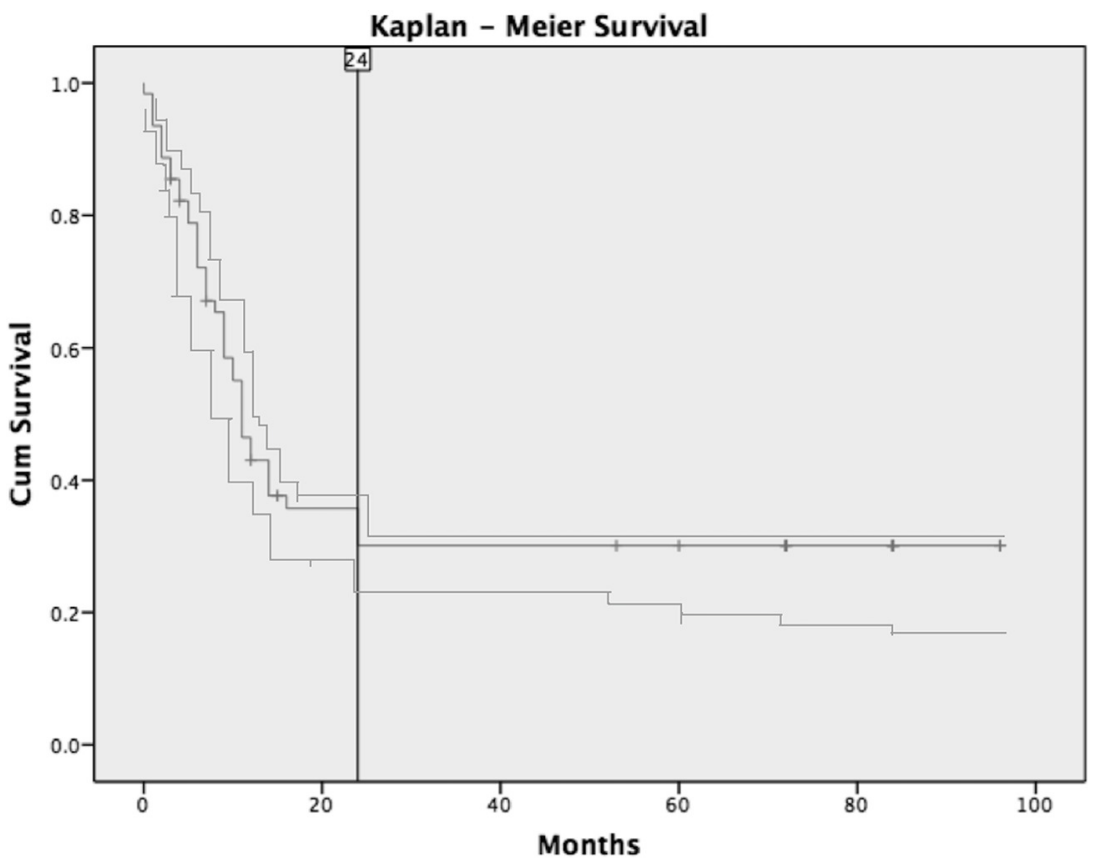

This study sought to offer prospective insights regarding the long-term impact of the unloader knee brace. While providing an indication of changes in patient utility from wearing the unloader brace, the two time-point analysis of the EQ-5D is unable to provide the full profile of utility variations across the duration of wear. In contrast, Dakin et $a l^{12}$ presented 5 years of EQ5D scores with values measured at baseline, 3 months, 12 months and yearly thereafter, demonstrating an approximate exponential relationship between utility and time since TKR. The findings from this study promote the potential value of the unloader knee brace and indicate further in-depth study would be beneficial. Increasing the number of time points over which data are collected should be considered a priority.

The effect of the knee brace would be more pronounced if compared with UKA or HTO as the cost utility is higher compared with TKR. The calculations used in this study are based on the 20-year average of a well-performing TKR, without considering the cost of subsequent revisions and complications of the expensive revision surgery.

Table 2 Mean EQ-5D and the QALYs gained results for all patients and duration of wear sub-groups

\begin{tabular}{lccccc}
\hline & \multicolumn{5}{c}{ Duration of wear (months) } \\
\hline & All & $1-6$ & $7-12$ & $13-24$ & $25+$ \\
Pre-EQ-5D & $0.012(0.194)$ & 0.000 & 0.068 & -0.003 & -0.030 \\
Post-EQ-5D & $0.432(0.291)$ & 0.415 & 0.511 & 0.373 & 0.391 \\
Difference EQ-5D & $0.420(0.354)$ & 0.415 & 0.443 & 0.376 & 0.420 \\
QALY Gain & $0.435(0.714)$ & 0.0631 & 0.176 & 0.268 & 1.292 \\
\hline
\end{tabular}

EQ-5D, EuroQol five dimensions; QALY, quality-adjusted life year. 
Table 3 Eight-month knee brace costs, QALYs gained and ICER

Costs (f)

(CI 95\%)
QALYs gained

(CI 95\%)
$\operatorname{ICER}(£)$

0.065 (0.045 to 0.086$)$

ICER, incremental cost-effectiveness ratio; QALY, quality-adjusted life year.

With regards to the unloading knee brace, according to patient feedback, $43 \%$ had soft tissue issues with the brace requiring technical support. Our findings are concurrent with published literature, which suggests that this is a result of the brace being fitted poorly. ${ }^{14}$ Therefore, a follow-up appointment at 1 month, 3 months and 6 months could give the opportunity for patients to attempt fitting the brace at home themselves accurately, acknowledge any skin irritation and change the fit accordingly. This would increase compliance and consequently increase the chances of success.

There was no significant difference in outcome or duration of wear as well as the final outcome between gender, age, BMI, socioeconomic status, leg or compartment. Therefore, all patients with UOA could benefit from wearing the brace. The mean BMI in this group is 30 . This is the same as the average BMI in TKR for men and women in the UK between 2003 and 2012, ${ }^{15}$ therefore it should not be used as a selection factor for either surgery or the use of the unloader brace.

The patients involved in this study have been diagnosed with end-stage arthritis and have been treated with analgesia, steroid and hyaluronan injections with unsatisfactory results. Due to the funding issues and the long waiting lists for surgical intervention in South
Wales, the unloader knee brace was initially used as a temporary device to help patients return to activity while waiting for surgery. Through this long-term follow-up, half of these patients were still functioning well with the brace and have been taken off the surgical intervention list. The primary focus of this study is based on economics and clinical outcome measures, as patients were reporting excellent outcomes at the longterm follow-up, radiological investigations were not justified.

Our study has provided long-term evidence for the use of unloader knee brace, however it has some limitations; it is a single-centre study with a small sample size, but all patients were followed up with a long-term average. Brace fitting also limited the study due to inconsistent fitting leading to compliance issues. A follow-up nurse-led clinic could improve long-term wear of the brace. Also, cost and time before treatment varies in different countries.

The current literature is uncertain of the long-term effects of the unloading brace. The primary end-point of this study is surgical intervention and that has shown to be improved by wearing the brace for the long term. The long duration of this study and its findings suggest the existence of long-term benefits of the unloader

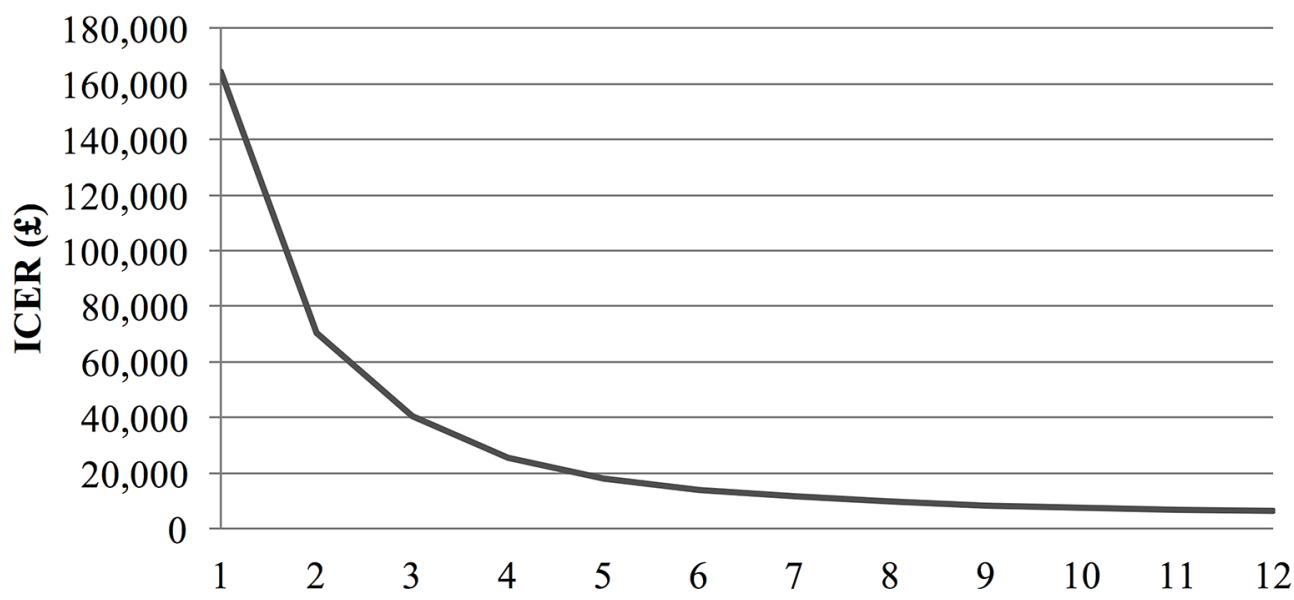

Duration of Wear (months)

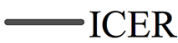

Figure 3 Incremental Cost-Effectiveness Ratio (ICER) exponentially decreases as the duration of wear increases. Compared with no treatment, the unloader knee brace can be considered cost-effective within the NICE threshold for duration of wear of 4 months or more. NICE, National Institute for Health and Care Excellence. 
Table 4 Full-term knee brace costs, QALYs gained and ICER

\begin{tabular}{|c|c|c|c|c|c|}
\hline & $\begin{array}{l}\text { Costs (£) } \\
(\mathrm{Cl} 95 \%)\end{array}$ & $\begin{array}{l}\text { QALYs gained } \\
\text { (CI 95\%) }\end{array}$ & $\begin{array}{l}\text { Change in Costs (£) } \\
\text { (Cl 95\%) }\end{array}$ & $\begin{array}{l}\text { Change in QALYs } \\
\text { (Cl 95\%) }\end{array}$ & $\begin{array}{l}\text { ICER } \\
\text { (f) }\end{array}$ \\
\hline $\begin{array}{l}\text { Unloader knee } \\
\text { brace }\end{array}$ & 625 (622 to 628) & 0.435 (0.255 to 0.615$)$ & $-123(-414$ to 168$)$ & $0.019(-0.103$ to 0.141$)$ & -6467 \\
\hline TKR & 749 (456 to 1041$)$ & 0.416 (0.252 to 0.581$)$ & & & \\
\hline
\end{tabular}

*Unloader knee brace dominates TKR.

ICER, incremental cost-effectiveness ratio; TKR, total knee replacement; QALY, quality-adjusted life year.

knee brace and advocate the need for further longterm research.

\section{Summary \\ Unloader knee brace}

- Six months of brace use halved the chance for surgery compared with 3 months or less use.

- Patients who wore the brace for 2 years or more did not require surgery at 8 years follow-up.

- Unloader knee brace has a similar QALY gain compared with TKR at 8 years follow-up (0.435).

- Short-term use of unloader knee brace can improve quality of life.

- It is cost-effective to use the unloader knee brace while patients are waiting for surgery.

\section{Conclusion}

The unloader knee brace can delay surgery and indeed deem it unnecessary over the long term for patients (39\%) with unicompartmental arthritis. Patients were able to return to their daily activities and work while using the unloader knee brace. The duration of wear was proportional to a successful outcome; we recommend education, motivation and support up to 24 months to increase compliance with its use. The unloader knee brace proposes a cost-effective nonoperative option for the treatment of unicompartmental knee osteoarthritis that can be quickly administered and significantly improve the patient's quality of life. Given that patients can self-select surgical intervention, the unloader knee brace has been shown to be cost-effective as either a complement, or alternative, to surgery. It is particularly useful in the younger age group $(<50)$, as delaying surgical intervention could reduce the demand for highly complex and expensive revision knee surgery in the future.

Collaborator WelshBone-South Wales Orthopaedics Research Network team: J Brock, DM Golding, C Perkins, W Abdul, RDJ Whitham, LE McMillan, AW Gardner and SJ Rozewicz.

Contributors PYFL and APC designed, planned the study and written the manuscript and revision. ERS conducted data collection, proof read the manuscript and revision. SRSH and TGW performed economic analysis, written the manuscript and revision. PYFL submitted the manuscript.

Funding Open access fees supported by WelshBone.
Competing interests None declared.

Patient consent Full dataset available from the corresponding author at leepyf@cardiff.ac.uk .

Ethics approval Hospital board approval.

Provenance and peer review Not commissioned; externally peer reviewed.

Data sharing statement Participants gave informed consent for data sharing and the presented data are anonymised and risk of identification is low.

Open Access This is an Open Access article distributed in accordance with the Creative Commons Attribution Non Commercial (CC BY-NC 4.0) license, which permits others to distribute, remix, adapt, build upon this work noncommercially, and license their derivative works on different terms, provided the original work is properly cited and the use is non-commercial. See: http:// creativecommons.org/licenses/by-nc/4.0/

(C) Article author(s) (or their employer(s) unless otherwise stated in the text of the article) 2017. All rights reserved. No commercial use is permitted unless otherwise expressly granted.

\section{REFERENCES}

1 Dakin H, Gray A, Fitzpatrick R, et al; The KAT Trial Group. Rationing of total knee replacement: a cost-effectiveness analysis on a large trial data set. BMJ Open 2012;2:e000332.

2 Sharma L, Song J, Felson DT, et al. The role of knee alignment in disease progression and functional decline in knee osteoarthritis. JAMA 2001;286:188-95.

3 Li CS, Bhandari M. Cost-effectiveness of unicompartmental knee arthroplasty, high tibial osteotomy, and KineSpring ${ }^{\circledR}$ knee implant system for unicompartmental osteoarthritis of the knee. J Long Term Eff Med Implants 2013;23:189-98.

4 Smith WB, Steinberg J, Scholtes S, et al. Medial compartment knee osteoarthritis: age-stratified cost-effectiveness of total knee arthroplasty, unicompartmental knee arthroplasty, and high tibial osteotomy. Knee Surgery, Sports Traumatology, Arthroscopy 2015

5 Wales N. Annual patient episode database wales (PEDW) Data Tables Wales 2016.

6 Wilson B, Rankin H, Barnes CL. Long-term results of an unloader brace in patients with unicompartmental knee osteoarthritis. Orthopedics 2011;34:e334-7.

7 Lorbach O, Mosser P, Pape D. [Sense or nonsense of unloader braces]. Orthopade 2014;43:984-7.

8 Duivenvoorden T, Brouwer RW, van Raaij TM, et al. Braces and orthoses for treating osteoarthritis of the knee. The Cochrane database of systematic reviews 2015;3:CD004020.

9 Össur. Unloader One: Össur UK. 2016. http://www.ossur.co.uk/oasolutions/oa-products/oa-knee/oa-knee-braces/unloader-one

10 EuroQol. EQ-5D-3L user guide: Basic user information on how to use the EQ-5D-3L instrument. 2015. http://www.euroqol.org/fileadmin/ user_upload/Documenten/PDF/Folders_Flyers/EQ-5D-3L_ UserGuide_2015.pdf5.1

11 Curtis L, Burns A. Unit costs of health and social care 2015. 2015 http://www.pssru.ac.uk/project-pages/unit-costs/2015/

12 Dakin H, Gray A, Fitzpatrick R, et al; KAT Trial Group. Rationing of total knee replacement: a cost-effectiveness analysis on a large trial data set. BMJ Open 2012;2:e000332.

13 National institute for clinical excellence. guide to the methods of technical appraisal. NICE 2013. https://www.nice.org.uk/process/ pmg9/chapter/foreword 
14 Squyer E, Stamper DL, Hamilton DT, et al. Unloader knee braces for osteoarthritis: do patients actually wear them? Clin Orthop Relat Res 2013;471:1982-91.
15 Registry NJ. NJR 10th annual report 2013. 2013. http://www. njrcentre.org.uk/njrcentre/NewsandEvents/NJR10 thAnnualReport2013/tabid/330/Default.aspx 